\title{
Superfluidity and Magnetism in Multicomponent Ultracold Fermions
}

\section{Citation}

Cherng, R. W., G. Refael, and E. Demler. 2007. "Superfluidity and Magnetism in Multicomponent Ultracold Fermions." Physical Review Letters 99 (13) (September 28). doi:10.1103/ physrevlett.99.130406.

\section{Published Version}

doi:10.1103/PhysRevLett.99.130406

\section{Permanent link}

http://nrs.harvard.edu/urn-3:HUL.InstRepos:27890259

\section{Terms of Use}

This article was downloaded from Harvard University's DASH repository, and is made available under the terms and conditions applicable to Other Posted Material, as set forth at http:// nrs.harvard.edu/urn-3:HUL.InstRepos:dash.current.terms-of-use\#LAA

\section{Share Your Story}

The Harvard community has made this article openly available.

Please share how this access benefits you. Submit a story.

Accessibility 


\title{
Superfluidity and Magnetism in Multicomponent Ultracold Fermions
}

\author{
R.W. Cherng, ${ }^{1}$ G. Refael, ${ }^{2}$ and E. Demler ${ }^{1}$ \\ ${ }^{1}$ Department of Physics, Harvard University, Cambridge, Massachusetts 02138, USA \\ ${ }^{2}$ Department of Physics, California Institute of Technology, Pasadena, California 91125, USA
}

(Received 2 May 2007; published 28 September 2007)

\begin{abstract}
We study the interplay between superfluidity and magnetism in a multicomponent gas of ultracold fermions. Ward-Takahashi identities constrain possible mean-field states describing order parameters for both pairing and magnetization. The structure of global phase diagrams arises from competition among these states as functions of anisotropies in chemical potential, density, or interactions. They exhibit first and second order phase transition as well as multicritical points, metastability regions, and phase separation. We comment on experimental signatures in ultracold atoms.
\end{abstract}

DOI: 10.1103/PhysRevLett.99.130406

Fermionic $s$-wave superfluidity requires pairing between fermions with different internal states. However, the nature of superfluidity for $N=2$ component systems and $N \geq 3$ differs fundamentally. Superfluidity is suppressed by magnetization for $N=2$ because there is one way to pair and not every particle can find a partner. This led to proposals for exotic paired states with broken translational symmetry $[1,2]$ or gapless excitations $[3,4]$. In contrast, superfluidity drives magnetization for $N \geq 3$. Condensation energy favors enhancing the populations for paired components to different degrees depending on the density of states and interaction energy. This occurs by cannibalizing the populations for unpaired components.

Ultracold atoms offer direct access to multicomponent fermionic superfluids. Observation of two component, equal population superfluids used Feshbach resonances to tune interactions [5-8]. The population for each component is both tunable and essentially conserved, enabling later experiments on superfluidity with imbalance $[9,10]$. Recently, scattering lengths and locations of overlapping Feshbach resonances between all three of the nearly degenerate lowest sublevels of ${ }^{6} \mathrm{Li}$ were measured [11]. This suggests experiments with $N \geq 3$ are within reach.

In this Letter, we consider the interplay of superfluidity and magnetism within a mean-field theory of $N \geq 3$ multicomponent fermions, each individually conserved. The fundamental question we address is which states out of the large space of order parameters describing both pairing $\Delta$ and magnetization $M$ are realized in physical systems. Our approach is based upon the derivation of a novel WardTakahashi (WT) identity [Eq. (6)] which provides fundamental constraints on possible mean-field states. We demonstrate this WT identity naturally leads to a specific form for the microscopic pairing wave function which we call diagonal pairing states (DPS) illustrated in Fig. 1. In these states, gapless excitations always exist for $N$ odd and are still possible for $N$ even.

Having classified the mean-field states, we derive global phase diagrams describing the competition among the DPS as shown in Figs. 2 and 3. There are several generic features of these phase diagrams which arise from the
PACS numbers: 05.30.Jp, 03.75.Mn, 03.75.Ss

structure of the DPS and general arguments. For $N \geq 3$, $\Delta \Delta^{\dagger}$ acts as an external field for $M$ through $M \Delta \Delta^{\dagger}$. This coupling vanishes identically on group theoretical grounds for $N=2$ where $M$ couples to $\Delta$ only at higher order. Pairing always drives magnetization for $N$ odd and generically does so for $N$ even through the coupling $M \Delta \Delta^{\dagger}$. First order transitions and corresponding metastability as well as phase separation regions separate different DPS while second order transitions separate DPS and the normal state. These transitions terminate at bicritical and multicritical points.

Previous theoretical works have also addressed superfluditiy with $N \geq 3$ components of fermions. This includes analysis of mean-field states for $N=3[12,13]$ and $N=4$ $[14,15]$, as well as phase diagrams for $N=3[16,17]$. We focus on classification of allowed pairing states through general symmetry arguments and Ward-Takahashi identities. This complementary approach allows us to obtain generic and robust features of both the resulting states and phase diagrams as well as providing a unified perspective on pairing in multicomponent fermionic systems.

We begin with the action $S(H)=S_{0}+S_{\text {int }}(\Gamma)$, where

$$
\begin{aligned}
S_{0}(H) & =\sum_{\alpha \beta}\left[\left(\partial_{\tau}+\frac{\nabla^{2}}{2 m}\right) \delta_{\alpha \beta}-H_{\alpha \beta}\right] \bar{\psi}_{\alpha} \psi_{\beta}, \\
S_{\text {int }}(\Gamma) & =-\sum_{\alpha \beta \gamma \delta} \Gamma_{\alpha \beta \gamma \delta} \bar{\psi}_{\alpha} \bar{\psi}_{\beta} \psi_{\gamma} \psi_{\delta},
\end{aligned}
$$
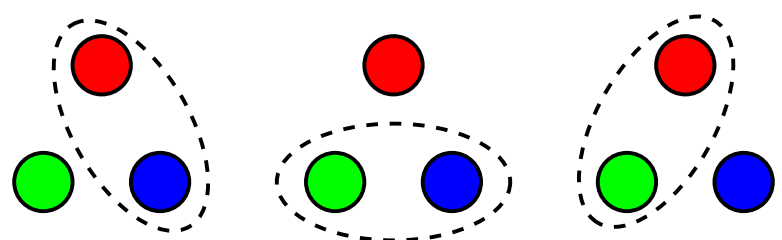

FIG. 1 (color online). Systematically pairing (ellipses) two components (colors) at a time generates the diagonal pairing states shown for $N=3$. These states satisfy the microscopic constraints imposed by Ward-Takahashi identities derived in the text, while linear combinations do not. Paired (unpaired) components have gapped (gapless) quasiparticle excitations. 
describing a dilute gas of fermions with mass $m$ and attractive contact interactions where $\psi_{\alpha}, \bar{\psi}_{\alpha}$ with $\alpha=$ $1 \ldots N$ are Grassman variables. The partition function is $Z(H, \Gamma)=\int \mathcal{D}[\bar{\psi}, \psi] \exp \left[-\int_{0}^{\beta} d \tau \int d^{D} \mathbf{x} S(H, \Gamma)\right], \quad \beta \quad$ is the inverse temperature, $D$ the dimensionality, and $k_{B}=$ $\hbar=1$. For a system with individually conserved components, $H_{\alpha \beta}=\mu_{\alpha} \delta_{\alpha \beta}, \Gamma_{\alpha \beta \gamma \delta}=\frac{1}{4}\left(\lambda_{\alpha \gamma}+\lambda_{\beta \delta}\right) \delta_{\alpha \delta} \delta_{\beta \gamma}-$ $(\gamma \leftrightarrow \delta)$, contributing $\mu_{\alpha} n_{\alpha}$ and $\lambda_{\alpha \beta} n_{\alpha} n_{\beta}$ to the action, where $n_{\alpha}=\bar{\psi}_{\alpha} \psi_{\alpha}$. Here $\mu_{\alpha}$ is a chemical potential while $\lambda_{\alpha \beta}$ describes interactions between fermionic densities.

The standard mean-field theory arises from a HubbardStratonovich transformation [18] which introduces bosonic pairing $\Delta_{\alpha \beta}$ and magnetization $M_{\alpha \beta}$ order parameters. They satisfy the saddle-point equations

$$
M_{\alpha \beta}=T g_{M} \sum_{\omega_{n}, \mathbf{k}} G_{\alpha \beta}, \quad \Delta_{\alpha \beta}=\frac{T g_{\Delta, \alpha \beta}}{2} \sum_{\omega_{n}, \mathbf{k}} F_{\alpha \beta},
$$

where $\omega_{n}=(2 n+1) \pi / \beta$ are Matsubara frequencies, $\mathbf{k}$ are momenta, $T$ is the temperature, and $\lambda_{\alpha \beta}=\frac{1}{4} g_{\Delta, \alpha \beta}-$ $\frac{1}{2} g_{M}\left(1+\frac{1}{N}\right)$ for decoupling interaction anisotropies in the pairing channel. Normal $G_{\alpha \beta}\left(i \omega_{n}, \mathbf{k}\right)$ and anomalous $F_{\alpha \beta}\left(i \omega_{n}, \mathbf{k}\right)$ Green's functions satisfy

$$
\left[\begin{array}{cc}
G & F^{\dagger} \\
F & -G^{\dagger}
\end{array}\right]=\left[\begin{array}{cc}
-\xi-H+M & \Delta^{\dagger} \\
\Delta & \xi^{\dagger}+H^{\dagger}-M^{\dagger}
\end{array}\right]^{-1},
$$

where $\xi_{\alpha \beta}=\left(i \omega_{n}-\mathbf{k}^{2} / 2 m\right) \delta_{\alpha \beta}$ in a matrix notation with suppressed indices. As matrices, $M^{\dagger}=M$ and $H^{\dagger}=$ $H$ are Hermitian while $\Delta^{T}=-\Delta$ is skew symmetric.

Having introduced the microscopic action and meanfield theory, we outline our two main results: the classification of allowed mean-field states through WT identities immediately following and the construction of mean-field phase diagrams following Eq. (9). Details will be presented elsewhere [19].

To derive WT identities, we consider field transformations under the unitary group $U(N)$ acting as

$$
\psi_{\alpha} \rightarrow \psi_{\alpha}^{\prime}=\sum_{\beta} U_{\alpha \beta} \psi_{\beta}, \quad \bar{\psi}_{\alpha} \rightarrow \bar{\psi}_{\alpha}^{\prime}=\sum_{\beta} \bar{\psi}_{\beta} U_{\beta \alpha}^{\dagger},
$$

with $U_{\alpha \beta}$ a unitary matrix. When viewed as a field redefinition, the coupling constants also transform $H_{\alpha \beta} \rightarrow H_{\alpha \beta}^{\prime}=\sum_{\gamma \delta} U_{\alpha \gamma} H_{\gamma \delta} U_{\delta \beta}^{\dagger}, \quad \Gamma_{\alpha \beta \gamma \delta} \rightarrow \Gamma_{\alpha \beta \gamma \delta}^{\prime}=$ $\sum_{\mu \nu \rho \sigma} U_{\alpha \mu} U_{\beta \nu} \Gamma_{\mu \nu \rho \sigma} U_{\rho \gamma}^{\dagger} U_{\sigma \delta}^{\dagger}$ in order to describe the same physical system. By definition, $Z(H, \Gamma)=Z\left(H^{\prime}, \Gamma^{\prime}\right)$ is invariant.

This invariance arises from a field redefinition and is not a physical symmetry. Anisotropies in $\mu_{\alpha}$ and $\lambda_{\alpha \beta}$ explicitly break $U(N) \rightarrow U(1)^{N}$ describing $N$ conserved densities in the normal state. The superfluid state spontaneously breaks $U(N) \rightarrow U(1)^{N} \rightarrow U(1)^{N-P}$, where $P$ is the number of nonzero pairing amplitudes.

However, it still gives a WT identity (see Ref. [20]) expressing $Z(H, \Gamma)=Z\left(H^{\prime}, \Gamma^{\prime}\right)$ under an infinitesimal transformation to first order

$$
\left(\mu_{\alpha}-\mu_{\beta}\right) \frac{\delta Z(H, \Gamma)}{\delta H_{\beta \alpha}}+\sum_{\gamma}\left(\lambda_{\alpha \gamma}-\lambda_{\gamma \beta}\right) \frac{\delta Z(H, \Gamma)}{\delta \Gamma_{\gamma \beta \alpha \gamma}}=0
$$

for arbitrary $\alpha, \beta$. Within the mean-field approximation, this microscopic WT identity becomes an explicit and simple constraint on $M$ and $\Delta$

$$
\left(\mu_{\alpha}-\mu_{\beta}\right) g_{M}^{-1} M_{\alpha \beta}+\sum_{\gamma}\left(g_{\Delta, \alpha \gamma}^{-1}-g_{\Delta, \gamma \beta}^{-1}\right) \Delta_{\alpha \gamma} \Delta_{\gamma \beta}^{\dagger}=0 .
$$

Our first main result is that by analyzing this constraint we are able to classify the allowed mean-field states which we denote DPS.

We first give a simple physical description of the DPS. The microscopic pairing wave function for the DPS is given by using a Bardeen-Cooper-Schrieffer (BCS) $s$-wave state to pair two and only two components at a time in all possible ways. The WT identity prohibits states given by linear combinations of the DPS. This gives a discrete set of mean-field states as illustrated for $N=3$ in Fig. 1.

Next we briefly discuss some additional properties of the DPS which require a more detailed analysis of their structure. This begins by diagonalizing the matrix order parameters

$$
M=\sum_{i=1}^{N} M_{i} \mathbf{u}_{i} \mathbf{u}_{i}^{\dagger}, \quad \Delta=\sum_{i=1}^{\lfloor N / 2\rfloor} \Delta_{i}\left(\mathbf{v}_{2 i-1} \mathbf{v}_{2 i}^{T}-\mathbf{v}_{2 i} \mathbf{v}_{2 i-1}^{T}\right),
$$

where $\lfloor x\rfloor$ is the floor function, $M_{i}\left(\Delta_{i}\right)$ are real (complex) eigenvalues, and $\mathbf{u}_{i}^{\dagger} \mathbf{u}_{j}=\mathbf{v}_{i}^{\dagger} \mathbf{v}_{j}=\delta_{i j}$ are orthonormal eigenvectors.

We now show the WT identity of Eq. (6) requires the eigenvectors to be of the form

$$
\mathbf{u}_{i, \alpha}=\mathbf{v}_{i, \alpha}=S_{i, \alpha},
$$

where $S_{i, \alpha}$ is a $N \times N$ permutation matrix with exactly one nonzero matrix element in each row and column. We denote these states the DPS. Consider the generic case when $\mu_{\alpha} \neq \mu_{\beta}, g_{\Delta, \alpha \beta}^{-1} \neq g_{\Delta, \gamma \delta}^{-1}$. For $N=3$, the DPS give all possible mean-field states. States not of DPS form do not satisfy Eq. (6). For $N>3$, the DPS give generic mean-field states. States not of the DPS form can in principle satisfy Eq. (6), but off-diagonal elements are generally overdetermined by the saddle-point equations implying nonzero values are inconsistent.

Notice $M$ is always diagonal as expected for the normal state where $U(1)^{N}$ symmetry of $N$ conserved densities prohibits mixing between different components. However, superfluidity spontaneously breaks $P$ factors of $U(1)$ through off-diagonal elements of $\Delta_{\alpha \beta}$ where $P$ is the number of nonzero $\Delta_{i}$. In addition, quasiparticle excitations are 
gapped for paired components and are gapless for unpaired components which remain a Fermi liquid. There are at most $[N / 2\rfloor$ nonzero $\Delta_{i}$ implying one component is always gapless for $N$ odd. For $N$ even, a fully gapped quasiparticle spectrum occurs only when all $\Delta_{i}$ are nonzero. References [12,13] obtained similar results for $N=3$.

After classifying the allowed mean-field states and discussing some of their properties, we turn to our second main result on mean-field phase diagrams. For simplicity, we construct phase diagrams in the weak-coupling or BCS regime near the superfluid transition for small anisotropies. However, we stress the preceding analysis of the DPS is general. We minimize the free energy

$$
\begin{aligned}
F= & \operatorname{Tr}\left[\frac{1-2 \hat{g}_{M} a}{2 \hat{g}_{M}} \delta \hat{M} \delta \hat{M}+b \frac{T-T_{c}^{\mathrm{SYM}}}{T_{c}^{\mathrm{SYM}}} \hat{\Delta}^{\dagger} \hat{\Delta}\right] \\
& +\operatorname{Tr}\left[c \delta \hat{M} \hat{\Delta} \hat{\Delta}^{\dagger}+d \hat{\Delta}^{\dagger} \hat{\Delta} \hat{\Delta}^{\dagger} \hat{\Delta}\right]+\sum_{\alpha} 2 a \delta \hat{\mu}_{\alpha} \delta \hat{M}_{\alpha \alpha} \\
& +\sum_{\alpha \beta}\left(\delta \hat{g}_{\Delta, \alpha \beta}^{-1}-c \frac{\delta \hat{\mu}_{\alpha}+\delta \hat{\mu}_{\beta}}{2}\right) \hat{\Delta}_{\alpha \beta}^{\dagger} \hat{\Delta}_{\beta \alpha}
\end{aligned}
$$

given by $F=-T \log Z$, where we take $\mu_{\alpha}=E_{F}+\delta \mu_{\alpha}$, $g_{\Delta, \alpha \beta}^{-1}=g_{\Delta}^{-1}+\delta g_{\Delta, \alpha \beta}^{-1}, \quad M_{\alpha \beta}=g_{M} n\left(E_{F}\right)+\delta M_{\alpha \beta}$ with $E_{F}$ the Fermi energy, $n\left(E_{F}\right)$ the free fermion density, and from here on, quantities with a hat are rescaled with respect to $T_{c}^{\mathrm{SYM}}$, the critical temperature without anisotropies. Here $a, b, c, d$ are Ginzburg-Landau parameters with $a$, $b, d \sim \hat{E}_{F}^{(D-2) / 2}$ proportional to the density of states at $\hat{E}_{F}$ describing particle-hole symmetric contributions. In contrast, $c \sim \hat{E}_{F}^{(D-4) / 2}$ for $D=2$ and $c \sim \hat{E}_{F}^{(D-4) / 2} \log \hat{E}_{F}$ for $D \neq 2$ is essentially proportional to the derivative of the density of states at $\hat{E}_{F}$ and describes particle-hole symmetry breaking contributions.

General symmetry considerations allow us to classify the terms in the free energy. $U(N)$ symmetric terms are under Tr, the matrix trace. Notice the $\delta \hat{M} \hat{\Delta} \hat{\Delta}^{\dagger}$ term where $\hat{\Delta} \hat{\Delta}^{\dagger}$ acts as an external field to $\delta \hat{M}$. On group theoretical grounds, this term is nonvanishing only for $N \geq 3$. Terms outside the trace explicitly break $U(N) \rightarrow U(1)^{N}$, including a term quadratic in $\hat{\Delta}$. The structure of global phase diagrams follows from these two terms.

The main consequence of the $\delta \hat{M} \hat{\Delta} \hat{\Delta}^{\dagger}$ term is that pairing drives magnetization. Physically, this occurs because a shift in chemical potentials described by $\hat{M}$ yields a gain in condensation energy described by $\hat{\Delta} \hat{\Delta}^{\dagger}$. This generically occurs unless $\hat{\Delta} \hat{\Delta}^{\dagger} \propto \mathbf{1}$ is particle-hole symmetric with 1 the identity matrix. Only when all components pair and $\left|\Delta_{i}\right|=\left|\Delta_{j}\right|$ does this occur. For $N$ odd, one component is always unpaired so magnetization always develops. For $N$ even, only when the $N / 2$ independent equations determining $\Delta_{i}$ give $\left|\Delta_{i}\right|=\left|\Delta_{j}\right|$ is it possible to have pairing without magnetization.

A similar situation occurs for $p$-wave pairing in ${ }^{3} \mathrm{He}$ or the organic superconductors. Unitary states describe pair- ing decoupled from magnetization while nonunitary states describe pairing coupled to magnetization [21]. Only a single constraint $\left|\mathbf{d} \times \mathbf{d}^{*}\right|=0$ on the $\mathbf{d}$-vector describing $p$-wave pairing is necessary for a unitary state. It is more difficult for the $N / 2$ independent equations determining $\Delta_{i}$ to give $\left|\Delta_{i}\right|=\left|\Delta_{j}\right|$ for the analog of unitary states in multicomponent $s$-wave pairing.

We now discuss the $N=3$ phase diagram in Fig. 2 with given interactions satisfying the generic condition $\delta \hat{g}_{\Delta, \alpha \beta}^{-1} \neq \delta \hat{g}_{\Delta, \gamma \delta}^{-1}$. We consider both fixed chemical potential $\hat{\mu}_{\alpha}$ and fixed particle density $n_{\alpha}$ as

$$
\begin{aligned}
\hat{\mu}_{\alpha} & =\hat{E}_{F} \mathbf{x}_{0, \alpha}+\hat{\mu}_{1} \mathbf{x}_{1, \alpha}+\hat{\mu}_{2} \mathbf{x}_{2, \alpha}, \\
n_{\alpha} & =n\left(E_{F}\right) \mathbf{x}_{0, \alpha}+n_{1} \mathbf{x}_{1, \alpha}+n_{2} \mathbf{x}_{2, \alpha},
\end{aligned}
$$

where $\quad \mathbf{x}_{0}=[1,1,1], \quad \mathbf{x}_{1}=[1,-1,0] / \sqrt{2}, \quad \mathbf{x}_{2}=$ $[1,1,-2] / \sqrt{6}$, and $\hat{\mu}_{i}, n_{i}$ parametrize anisotropies.

First consider fixed $\hat{\mu}_{\alpha}$ (top Fig. 2). When $T>T_{c}^{\text {SYM }}$, small anisotropy favors the normal state. Increasing anisotropy favors pairing by increasing the density of states for some components at the expense of others. This drives a second order transition into one of the three DPS. Tuning the direction of the anisotropy drives first order transitions between DPS when two of these states are degenerate along lines of enhanced symmetry. First order lines terminate at bicritical points from which metastability regions, where an additional DPS is locally stable, branch out. This is the expected behavior for quadratic symmetry breaking $[22,23]$. Bicritical points terminate at the $U(3)$ symmetric multicritical point when $T=T_{c}^{\mathrm{SYM}}$. Below $T_{c}^{\mathrm{SYM}}$, first
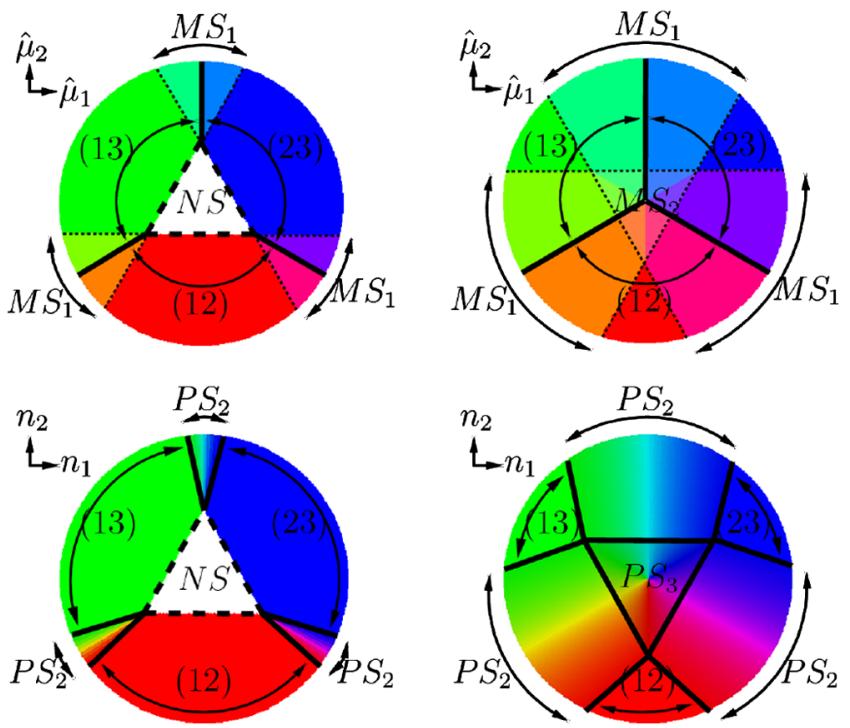

FIG. 2 (color online). $\quad N=3$ phase diagrams for $T>T_{c}^{\mathrm{SYM}}$ (left) and $T<T_{c}^{\mathrm{SYM}}$ (right) against anisotropies in chemical potential $\hat{\mu}_{\alpha}$ (top) and density $n_{\alpha}$ (bottom). $N S$ denotes the normal state and $(\alpha \beta)$ a state with $\alpha, \beta$ paired. Solid (dashed) lines are first (second) order transitions while dotted lines are boundaries for metastability regions. $M S_{i}$ denotes regions with $i$ additional metastable paired states and $P S_{i}$ a phase-separated mixture of $i$ paired states. 


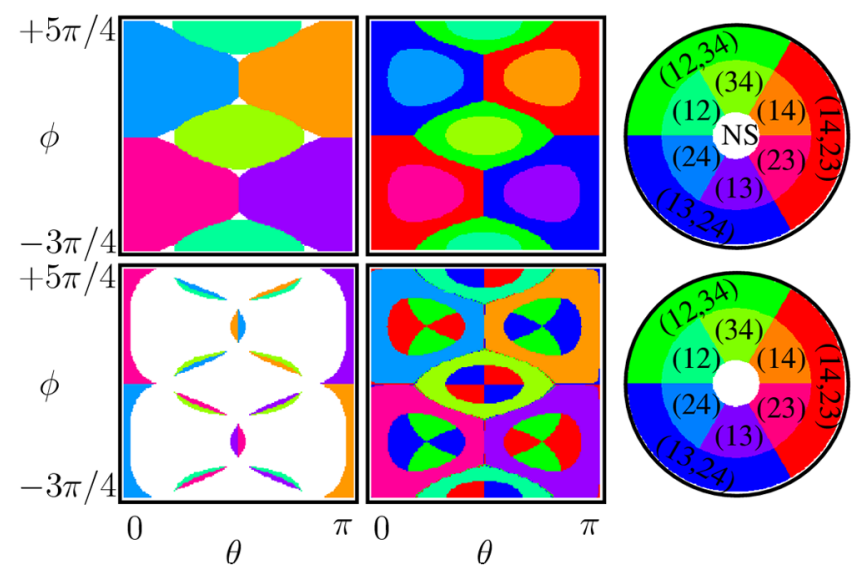

FIG. 3 (color online). Representative $N=4$ phase diagrams for $T>T_{c}^{\mathrm{SYM}}$ (left) and $T<T_{c}^{\mathrm{SYM}}$ (middle) against anisotropies in chemical potential $\mu_{\alpha}$ with legend (right) for the global minimum (top) and first metastable state (bottom). $\left(\alpha_{i} \beta_{i}, \ldots\right)$ denotes $\alpha_{i}, \beta_{i}$ paired and white regions $N S$ the normal state (top) or absence of metastable states (bottom). First (second) order transitions separate different paired states (paired states from the normal state).

order lines separate DPS and a region near small anisotropy where all three DPS are (meta)stable appears.

The Maxwell construction gives fixed $n_{\alpha}$ phase diagrams (bottom Fig. 2) from those for fixed $\hat{\mu}_{\alpha}$. The compressibility tensor $\kappa_{\alpha \beta} \propto-\partial^{2} F / \partial \hat{\mu}_{\alpha} \partial \hat{\mu}_{\beta}$ at fixed $\hat{\mu}_{\alpha}$ has positive eigenvalues except at boundaries between DPS. Here, $\hat{\Delta} \hat{\Delta}^{\dagger}$ and thus $\delta \hat{M}$ jump discontinuously. First order lines at fixed $\hat{\mu}_{\alpha}$ when two DPS are degenerate expand into phase-separated mixtures of those two states at fixed $n_{\alpha}$. For $T<T_{c}^{\mathrm{SYM}}$, the zero anisotropy point where all three DPS are degenerate expands into a phase-separated mixture of those three states at fixed $n_{\alpha}$.

Now consider the $N=4$ phase diagram in Fig. 3 with fixed chemical potential given by

$$
\begin{aligned}
\hat{\mu}_{\alpha}= & \hat{E}_{F} \mathbf{y}_{0, \alpha}+\hat{\mu}_{0} \cos (\theta) \mathbf{y}_{1, \alpha}+\hat{\mu}_{0} \sin (\theta) \cos (\phi) \mathbf{y}_{2, \alpha} \\
& +\hat{\mu}_{0} \sin (\theta) \sin (\phi) \mathbf{y}_{3, \alpha},
\end{aligned}
$$

where $\quad \mathbf{y}_{0}=[1,1,1,1], \quad \mathbf{y}_{1}=[1,-1,0,0] / \sqrt{2}, \quad \mathbf{y}_{2}=$ $[1,1,-2,0] / \sqrt{6}, \mathbf{y}_{3}=[1,1,1,-3] / \sqrt{12}$, and $\theta, \phi$ parametrize the anisotropies.

For $N>3$, condensation energy favors development of more pairing amplitudes as $T$ is lowered. The normal state competes with the six DPS with only one pairing amplitude for $T>T_{c}^{\mathrm{SYM}}$ (top right Fig. 3). However, the three DPS with two pairing amplitudes and all components paired eventually dominate the phase diagram for $T<T_{c}^{\mathrm{SYM}}$ (top middle Fig. 3). Phase diagrams still exhibit second order transitions from the normal state to DPS and first order transitions between DPS.

We now comment on trapping effects and detection methods for applications to ultracold atoms. In the large particle number BCS regime, the local density approximation accurately maps phase diagrams at fixed chemical potential to phase diagrams with trapping. DPS are distinguished by both densities and pairing amplitudes for different components. State-selective imaging of density distributions $[9,10]$ and radio-frequency spectroscopy of the pairing gap [24] can be used to detect signatures of the various paired states.

In summary, we have studied the essential role magnetism plays in superfluidity of multicomponent fermions. By analyzing constraints imposed by Ward-Takahashi identities, we classified the allowed mean-field pairing states with both magnetization and pairing order parameters and used them to construct global phase diagrams. These phase diagrams have a rich structure with first and second order transitions meeting at multicritical points as well as metastability and phase-separated regions. We discussed applications to ultracold fermions.

We acknowledge useful discussions with Walter Hofstetter. This work was supported by NSF grant No. DMR-0132874, Harvard-MIT CUA, AFOSR, and NDSEG.

[1] P. Fulde and R. A. Ferrell, Phys. Rev. 135, A550 (1964).

[2] A. J. Larkin and Y. N. Ovchinnikov, Zh. Eksp. Teor. Fiz. 47, 1136 (1964) [Sov. Phys. JETP 20, 762 (1965)].

[3] G. Sarma, J. Phys. Chem. Solids 24, 1029 (1963).

[4] W. V. Liu and F. Wilczek, Phys. Rev. Lett. 90, 047002 (2003).

[5] C. A. Regal et al., Phys. Rev. Lett. 92, 040403 (2004).

[6] M. Zwierlein et al., Phys. Rev. Lett. 92, 120403 (2004).

[7] M. Bartenstein et al., Phys. Rev. Lett. 92, 120401 (2004).

[8] J. Kinast et al., Phys. Rev. Lett. 92, 150402 (2004).

[9] M. W. Zwierlein et al., Science 311, 492 (2006).

[10] G. B. Partridge et al., Science 311, 503 (2006).

[11] M. Bartenstein et al., Phys. Rev. Lett. 94, 103201 (2005).

[12] C. Honerkamp and W. Hofstetter, Phys. Rev. Lett. 92, 170403 (2004).

[13] C. Honerkamp and W. Hofstetter, Phys. Rev. B 70, 094521 (2004).

[14] C. Wu et al., Phys. Rev. Lett. 91, 186402 (2003).

[15] C. Wu, Mod. Phys. Lett. B 20, 1707 (2006).

[16] A. Rapp et al., Phys. Rev. Lett. 98, 160405 (2007).

[17] T. Paananen et al., Phys. Rev. A 73, 053606 (2006).

[18] J.W. Negele and H. Orland, Quantum Many-Particle Systems (Addison-Wesley, New York, 1988).

[19] R. W. Cherng, G. Refael, and E. Demler (to be published).

[20] J. Zinn-Justin, Quantum Field Theory and Critical Phenomena (Oxford University, Oxford, 1996).

[21] A. J. Leggett, Rev. Mod. Phys. 47, 331 (1975).

[22] D. R. Nelson and E. Domany, Phys. Rev. B 13, 236 (1976).

[23] D. J. Amit and Y. Y. Goldschmidt, Ann. Phys. (Leipzig) 114, 356 (1978).

[24] C. Chin et al., Science 305, 1128 (2004). 